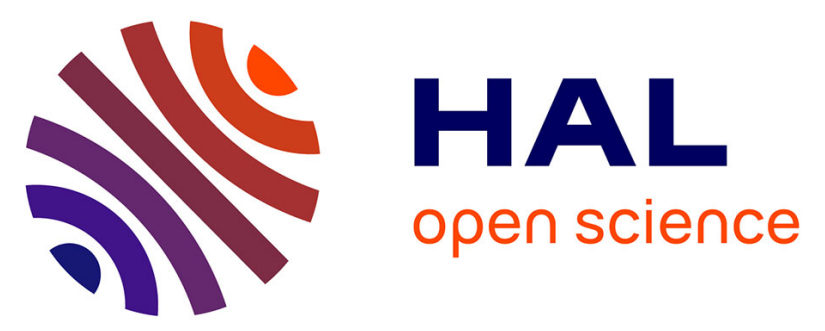

\title{
Détermination des grandeurs caractéristiques des analyseurs électrostatiques toriques. Application à l'optimisation d'analyseurs utilisés en physique spatiale
}

Pierrette Décréau, R. Prange, Jean-Jacques Berthelier

\section{- To cite this version:}

Pierrette Décréau, R. Prange, Jean-Jacques Berthelier. Détermination des grandeurs caractéristiques des analyseurs électrostatiques toriques. Application à l'optimisation d'analyseurs utilisés en physique spatiale. Revue de Physique Appliquée, 1972, 7 (2), pp.95-106. 10.1051/rphysap:019720070209500 . jpa-00243611

\section{HAL Id: jpa-00243611 \\ https://hal.science/jpa-00243611}

Submitted on 1 Jan 1972

HAL is a multi-disciplinary open access archive for the deposit and dissemination of scientific research documents, whether they are published or not. The documents may come from teaching and research institutions in France or abroad, or from public or private research centers.
L'archive ouverte pluridisciplinaire HAL, est destinée au dépôt et à la diffusion de documents scientifiques de niveau recherche, publiés ou non, émanant des établissements d'enseignement et de recherche français ou étrangers, des laboratoires publics ou privés. 
Classification

Physics abstracts

$06.10-06.20-20.50$

\title{
DÉTERMINATION DES GRANDEURS CARACTÉRISTIQUES DES ANALYSEURS ÉLECTROSTATIQUES TORIQUES APPLICATION A L'OPTIMISATION D'ANALYSEURS UTILISÉS EN PHYSIQUE SPATIALE
}

\author{
P. DECREAU
}

Groupe de Recherches Ionosphériques, CNRS, 45-Orléans 02

R. PRANGE

Laboratoire de Physique Stellaire et Planétaire, CNRS, 91-Verrières-le-Buisson

\author{
et J. J. BERTHELIER
}

Groupe de Recherches Ionosphériques, CNRS, 94-Saint-Maur-des-Fossés

(Reçu le 13 septembre 1971, révisé le 9 février 1972)

\begin{abstract}
Résumé. - On présente une méthode numérique de détermination des caractéristiques d'analyseurs électrostatiques de particules, valable dans le cas général d’électrodes toriques. Le champ électrostatique est exprimé sous forme d'un développement limité en fonction de coordonnées réduites, définissant la position d'un point par rapport à l'équipotentielle moyenne. On évalue les coefficients de ce développement jusqu'à un ordre donné quelconque, et la trajectoire d'une particule est déterminée par intégration numérique des équations du mouvement. On aboutit, par exploration des différents cas d'incidence, aux grandeurs caractéristiques de l'analyseur. Les résultats sont comparés avec ceux obtenus au moyen d'une simulation en cuve rhéographique.

On présente deux exemples concrets d'utilisation du programme numérique mis au point, exemples qui répondent à des problèmes de mesure en milieu spatial : analyseur à grands pouvoirs de résolution en énergie et en angle, analyseur à grand domaine d'entrée et large bande passante en énergie. La forme torique permet d'élargir de manière très appréciable le cadre des performances obtenues à l'aide d'analyseurs de formes classiques, cylindriques ou sphériques.

Abstract. - We present a numerical method to calculate the characteristic properties of electrostatic analysers in the general case of toroïdal plates. The electrostatic field is given by a series expansion of the reduced coordinates which gives the position of a point with respect to the mean trajectory. We calculate the coefficients of this series to the desired order, and the trajectory of a particle is then determined by integrating numerically the equations of motion. Investigating every case of incidence, we obtain the characteristic properties of the analyser. Results are compared with those obtained by a rheographic simulation of the analyser.

Two examples are given in which numerical programs were used. These examples correspond to typical problems in the earth environment : analysers with good resolution, in energy and angle, analysers with large entry area and wide energy range. The choice of a toroïdal shaped analyser improves considerably the performance obtained classically by using cylindrical or spherical analysers.
\end{abstract}

1. Introduction. - L'analyse et la détection des particules chargées de faible énergie, de quelques $\mathrm{eV}$ à $100 \mathrm{keV}$ environ, représentent un problème expérimental que l'on rencontre fréquemment en physique atomique et nucléaire ou en spectrométrie de masse par exemple. Le développement récent et rapide de la physique spatiale, en faisant apparaître de nouveaux objectifs et de nouvelles contraintes, a provoqué une évolution et des progrès considérables des techniques utilisées.
A bord des fusées et satellites, les dispositifs conventionnels, tels que les compteurs à scintillations et les détecteurs à semi-conducteurs associés à des analyseurs de hauteur d'impulsions, sont limités à des énergies de quelques dizaines de $\mathrm{keV}$ (seuil fixé par la traversée du dépôt métallique protecteur). En outre, au voisinage de ce seuil, la résolution des compteurs à scintillateurs est très mauvaise [1].

Depuis la mise au point des multiplicateurs d'électrons tubulaires, il est possible d'effectuer des mesures 
dans la gamme de quelques $\mathrm{eV}$ à quelques dizaines de $\mathrm{keV}$. Ces détecteurs, non sélectifs, sont alors précédés d'analyseurs électrostatiques ou magnétiques. Pour les énergies inférieures à $100 \mathrm{keV}$, et dans le cas d'expériences spatiales, les analyseurs électrostatiques sont préférables aux analyseurs magnétiques, car ils sont beaucoup moins lourds, moins encombrants et un seul dispositif peut explorer une très grande gamme d'énergie. Ils permettent, en outre, l'analyse des ions dont la rigidité magnétique est très grande. De tels dispositifs, très souples d'emploi et bien adaptés à une étude assez complète des flux particulaires de basse énergie de l'environnement terrestre, ont été largement utilisés. On peut citer Frank [2], Hoffman [3], Rème [4].

Les analyseurs électrostatiques cylindriques ou sphériques ont été étudiés par de nombreux auteurs, notamment Hughes et Rojansky [5], Clarke [6], Marmet et Kervin [7], Paolini et Theodoridis [8] à [10], Kerbele et Lassettre [11], Rogers [12], Ritchie [13], Minkov [14]. Ces deux types d'analyseurs représentent des cas particuliers d'une forme plus générale, l'analyseur torique.

Nous avons entrepris l'étude théorique de ce cas général, afin de déterminer les grandeurs caractéristiques (réponse angulaire, facteur de transmission, résolution en énergie), en fonction des paramètres géométriques de l'instrument.

2. Définition des paramètres géométriques et des grandeurs caractéristiques d'un analyseur torique. 2.A PARAmètres gÉOMÉTRIQUES. - L'analyseur est de révolution autour de l'axe $z z^{\prime}$ et a pour plan de symétrie le plan $z=0$ passant par $\mathrm{O}$; on considère seulement le cas où les cercles $(\mathrm{Ca})$ et $(\mathrm{Cb})$ ont même centre $\mathbf{O}^{\prime}$. Les différents paramètres géométriques sont définis sur la figure 1.

La collimation est assurée par une fente de largeur $d_{\mathrm{f}}$, parallèle au plan d'entrée, située à une distance $h$ de celui-ci, et telle que la normale au plan d'entrée fasse un angle $\alpha_{\mathrm{m}}$ avec l'axe de visée $C C_{\mathrm{f}}$.

On néglige tout effet de bord dans le volume engendré par la rotation de $S$, surface d'entrée, autour de $z^{\prime} z$. On définit :

- la distance interélectrode, $d=r_{\mathrm{a}}-r_{\mathrm{b}}$;

- les rayons de courbure moyens, $r_{\mathrm{c}}=\left(r_{\mathrm{a}}+r_{\mathrm{b}}\right) / 2$ et $R_{\mathrm{c}}=\left(R_{\mathrm{a}}+R_{\mathrm{b}}\right) / 2$;

- la hauteur curviligne, $s=R_{\mathrm{c}} \omega$.

Lorsqu'on calculera les grandeurs caractéristiques de l'ensemble analyseur-détecteur, ce dernier sera représenté par un cercle de diamètre $\sigma$, situé dans le plan de sortie, centré sur l'équipotentielle moyenne $T$ $\mathrm{du}$ plan de symétrie $\left(z=0, r=r_{\mathrm{c}}\right)$. Une fente de hauteur $z_{h}$ en réduit éventuellement la surface utile.

2. B GRANDEURS CARACTÉRISTIQUeS. - Les particules incidentes sont définies par :

- leur masse $M$, leur charge $q$, leur énergie $U_{0}$ (en $\mathrm{eV}$ ) ; on suppose les particules non relativistes,
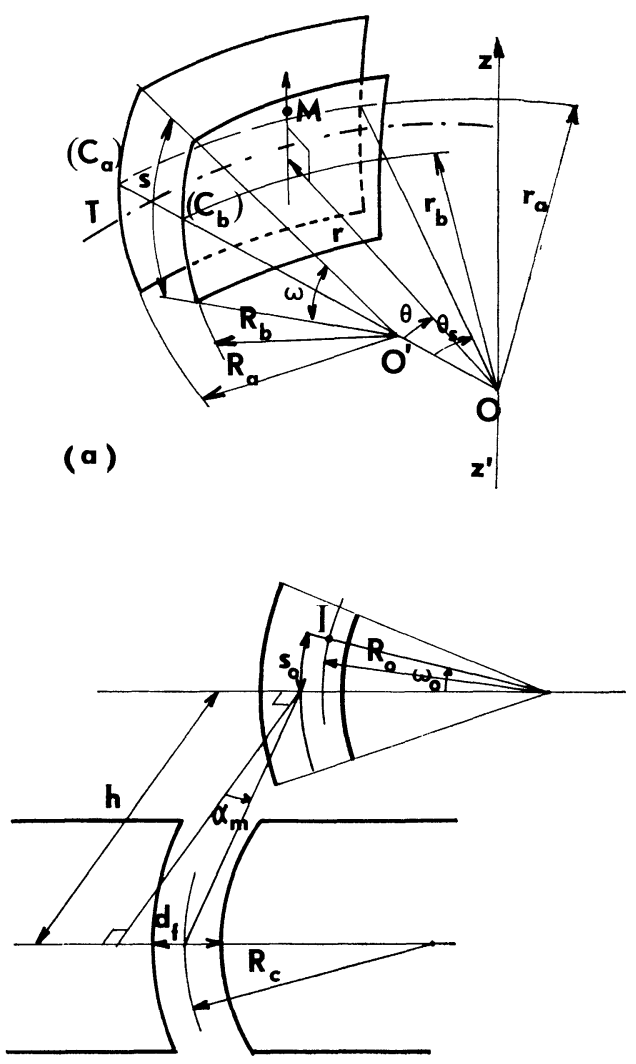

(b)

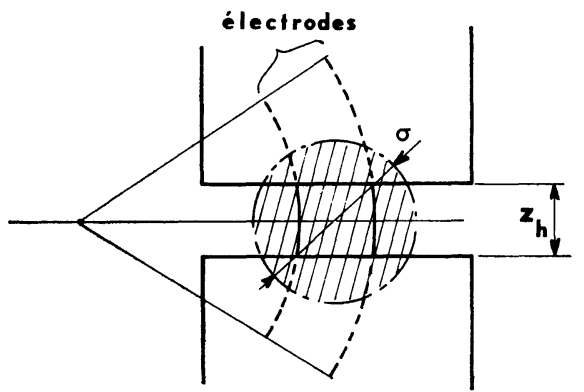

(c)

Fig. 1. - Géométrie de l'analyseur.

a) Forme des électrodes.

b) Plan d'entrée des électrodes et fente de collimation.

c) Plan de sortie des électrodes. La partie hachurée représente la surface détectrice d'un multiplicateur tubulaire d'électrons.

- leur direction, repérée par les angles $\alpha_{0}$ et $\varphi_{0}$ (Fig. 2).

L'intensité du flux incident sur la surface $S$ (exprimée en particules $\left.\mathrm{cm}^{-2} \cdot \mathrm{sr}^{-1} \cdot \mathrm{eV}^{-1} \cdot \mathrm{s}^{-1}\right)$ est alors décrite par une fonction $J\left(\alpha_{0}, \varphi_{0}, U_{0}\right)$ supposée uniforme étant donné les faibles dimensions de $S$.

Considérons les particules d'énergie $U_{0}$, arrivant sur la surface $S$ suivant une direction incidente comprise dans l'angle solide élémentaire $\mathrm{d} \Omega$ autour de la direction $\left(\alpha_{0}, \varphi_{0}\right)$. Une portion $n\left(\alpha_{0}, \varphi_{0}, U_{0}\right) \mathrm{d} \Omega$ de ces particules atteindra le détecteur. Elle a été reçue sur une partie $\Delta S\left(\alpha_{0}, \varphi_{0}, U_{0}\right)$ de la surface $S$ 


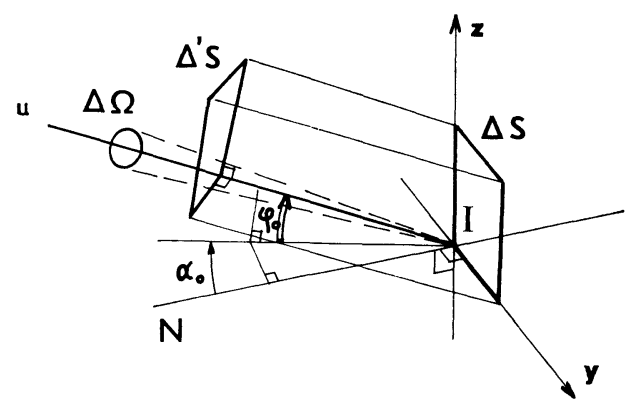

FIG. 2. - Iu : direction d'incidence.

IN : normale au plan d'entrée des électrodes, $y I z$.

(Fig. 2), dont la projection normale à la direction d'incidence est :

$\Delta^{\prime} S\left(\alpha_{0}, \varphi_{0}, U_{0}\right)=\Delta S\left(\alpha_{0}, \varphi_{0}, U_{0}\right) \cos \alpha_{0} \cos \varphi_{0}$.

Comme d'autre part :

$$
\mathrm{d} \Omega=\cos \varphi_{0} \mathrm{~d} \varphi_{0} \mathrm{~d} \alpha_{0},
$$

on peut écrire :

$n\left(\alpha_{0}, \varphi_{0}, U_{0}\right) \mathrm{d} \Omega=$

$=J\left(\alpha_{0}, \varphi_{0}, U_{0}\right) \Delta S\left(\alpha_{0}, \varphi_{0}, U_{0}\right) \cos \alpha_{0} \cos ^{2} \varphi_{0} \mathrm{~d} \alpha_{0} \mathrm{~d} \varphi_{0}$.

Les performances de l'analyseur imposent aux grandeurs $\alpha_{0}, \varphi_{0}$ et $U_{0}$ les bornes $\alpha^{\prime}, \alpha^{\prime \prime}, \varphi^{\prime}, \varphi^{\prime \prime}, U^{\prime}, U^{\prime \prime}$ (repérées sur la figure 3) qui définissent un domaine $D_{0}$ dans l'espace $\left(\alpha_{0}, \varphi_{0}, U_{0}\right)$ en dehors duquel aucune particule n'atteint le détecteur.
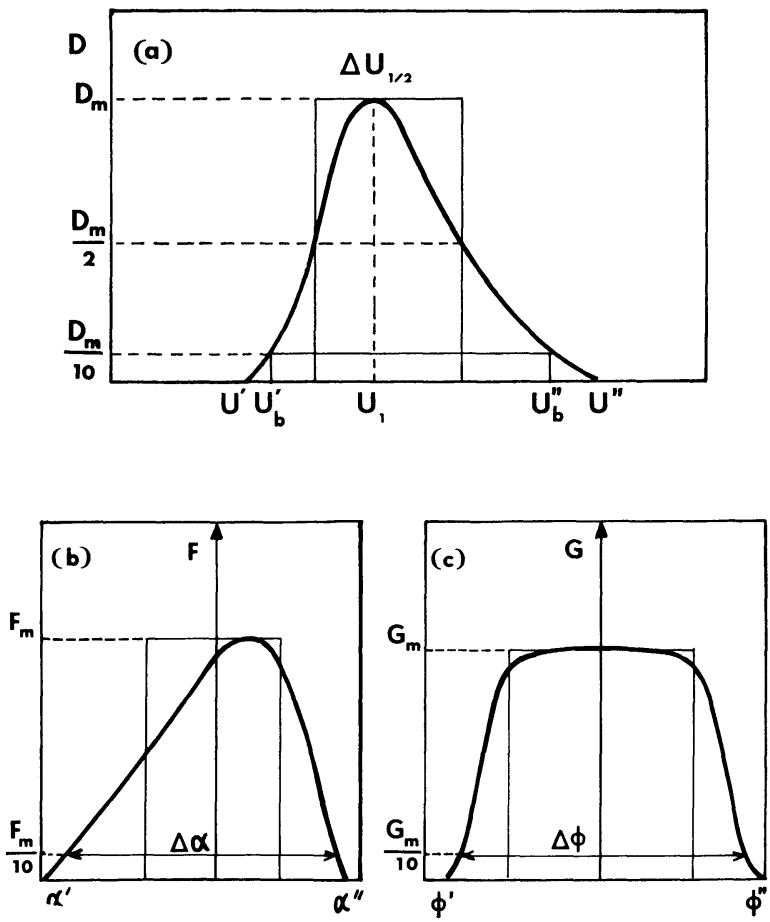

Fig. 3. - Courbes de réponse :

a) en énergie,

$b$ ) et $c$ ) : suivant les angles d'incidence.

: analyseur théorique idéal.
Le taux de comptage pour l'ensemble du domaine $D_{0}$ est alors :

$$
N=\int_{D_{0}} n\left(\alpha_{0}, \varphi_{0}, U_{0}\right) \Delta D_{0}
$$

ce qui donne :

$$
\begin{array}{r}
N=\int_{\alpha^{\prime}}^{\alpha^{\prime \prime}} \int_{\varphi^{\prime}}^{\varphi^{\prime \prime}} \int_{U^{\prime}}^{U^{\prime \prime}} J\left(\alpha_{0}, \varphi_{0}, U_{0}\right) \Delta S\left(\alpha_{0}, \varphi_{0}, U_{0}\right) \times \\
\quad \times \cos \alpha_{0} \cos ^{2} \varphi_{0} \mathrm{~d} \alpha_{0} \mathrm{~d} \varphi_{0} \mathrm{~d} U_{0} .
\end{array}
$$

En supposant le flux incident isotrope et indépendant de l'énergie, et en posant $J\left(\alpha_{0}, \varphi_{0}, U_{0}\right) \equiv 1$ sur le domaine $D_{0}$, on introduit les fonctions suivantes qui permettent de définir les caractéristiques de l'analyseur (Fig. 3) :

$$
\begin{aligned}
& D\left(U_{0}\right)= \\
& \quad=\int_{\alpha^{\prime}}^{\alpha^{\prime \prime}} \int_{\varphi^{\prime}}^{\varphi^{\prime \prime}} \Delta S\left(\alpha_{0}, \varphi_{0}, U_{0}\right) \cos ^{2} \varphi_{0} \cos \alpha_{0} \mathrm{~d} \alpha_{0} \mathrm{~d} \varphi_{0} \\
& F\left(\alpha_{0}\right)= \\
& \quad=\int_{\varphi^{\prime}}^{\varphi^{\prime \prime}} \int_{U^{\prime}}^{U^{\prime \prime}} \Delta S\left(\alpha_{0}, \varphi_{0}, U_{0}\right) \cos ^{2} \varphi_{0} \cos \alpha_{0} \mathrm{~d} \varphi_{0} \mathrm{~d} U_{0} \\
& G\left(\varphi_{0}\right)=\int_{\varphi}^{\varphi^{\prime \prime}} \int_{U^{\prime}}^{U^{\prime \prime}} \Delta S\left(\alpha_{0}, \varphi_{0}, U_{0}\right) \cos ^{2} \varphi_{0} \cos \alpha_{0} \mathrm{~d} \alpha_{0} \mathrm{~d} U_{0} .
\end{aligned}
$$

Remarquons au passage que :

$$
\int_{U^{\prime}}^{U^{\prime \prime}} D\left(U_{0}\right) \mathrm{d} U_{0}=\int_{\alpha^{\prime}}^{\alpha^{\prime \prime}} F\left(\alpha_{0}\right) \mathrm{d} \alpha_{0}=\int_{\varphi^{\prime}}^{\varphi^{\prime \prime}} G\left(\varphi_{0}\right) \mathrm{d} \varphi_{0} .
$$

On désigne par $U_{0 \text { n }}$ l'énergie nominale, c'est-à-dire l'énergie d'une particule qui arrive en incidence normale $\left(\alpha_{0}=0, \varphi_{0}=0\right)$ et dont la trajectoire est l'équipotentielle moyenne $T$ du plan de symétrie; la figure 3 indiquant la signification des grandeurs caractéristiques $U_{1}, D_{1}, D_{\mathrm{m}}, \Delta U_{1 / 2}, U_{\mathrm{b}}^{\prime}, U_{\mathrm{b}}^{\prime \prime}, \Delta \alpha$ et $\Delta \varphi$.

On définit également :

- la résolution en énergie et le pouvoir de résolution :

- à mi-hauteur :

$$
R_{\mathrm{u}}=\frac{\Delta U_{1 / 2}}{U_{1}}, \quad P_{\mathrm{r}}=\frac{1}{R_{\mathrm{u}}}
$$

- à la base :

$$
R_{\mathrm{ub}}=\frac{U_{\mathrm{b}}^{\prime \prime}-U_{\mathrm{b}}^{\prime}}{U_{1}}, \quad P_{\mathrm{rb}}=\frac{1}{R_{\mathrm{ub}}}
$$

- le facteur de géométrie moyen :

$$
\bar{g}=\frac{1}{\Delta U_{1 / 2}} \int_{U_{1}-1 / 2 \Delta U_{1 / 2}}^{U_{1}+1 / 2 \Delta U_{1 / 2}} D\left(U_{0}\right) \mathrm{d} U_{0} ;
$$


- le facteur de géométrie parasite :

$$
\begin{aligned}
g_{\mathrm{p}} & =\frac{1}{\left(U_{1}-\frac{1}{2} \Delta U_{1 / 2}\right)-U^{\prime}} \int_{U^{\prime}}^{U_{1}-1 / 2 \Delta U_{1 / 2}} D\left(U_{0}\right) \mathrm{d} U_{0} \\
& +\frac{1}{U^{\prime \prime}-\left(U_{1}+\frac{1}{2} \Delta U_{1 / 2}\right)} \int_{U_{1}+1 / 2 \Delta U_{1 / 2}}^{U^{\prime \prime}} D\left(U_{0}\right) \mathrm{d} U_{0}
\end{aligned}
$$

- le facteur de forme :

$$
f=\frac{g_{\mathrm{p}}}{\bar{g}+g_{\mathrm{p}}}
$$

- le domaine en énergie :

$$
C=D_{\mathrm{m}} R_{\mathrm{u}}
$$

- l'ouverture angulaire :

$$
A=\max (\Delta \alpha, \Delta \varphi) \text {. }
$$

Sur la figure 3 , on a tracé en outre, en traits fins, les courbes D, F, G correspondant à un « détecteur idéal ", dont la transmission serait indépendante de $\alpha_{0}, \varphi_{0}, U_{0}$ sur tout le domaine $D_{0}$ (dans ce cas, $g_{\mathrm{p}}=0$, $f=0$ ). On essaiera d'obtenir des courbes de réponse s'approchant le plus possible de cette forme.

3. Méthodes d'analyse numérique. - Les fonctions que nous cherchons à déterminer sont $D\left(U_{0}\right), F\left(\alpha_{0}\right)$, $G\left(\varphi_{0}\right)$. En pratique, la résolution de ce problème nous conduit à calculer la trajectoire de chaque particule incidente, et à rechercher si cette particule peut atteindre le détecteur sans avoir au préalable franchi les surfaces qui définissent le volume utile de l'analyseur.

On peut voir que l'énergie $U_{0}$ d'une part, et le produit $q V$ de la charge de la particule par la d. d. p. appliquée entre les électrodes d'autre part, sont homogènes (dimension $\mathrm{ML}^{2} \mathrm{~T}^{-2}$ ). Toutes conditions égales par ailleurs, la trajectoire d'une particule $\left(U_{0}, q\right)$ dans le champ (grad $V$ ) sera identique à celle d'une particule $\left(U_{0}^{\prime}, q^{\prime}\right)$ telle que $U_{0}^{\prime} / q^{\prime}=k\left(U_{0} / q\right)$ dans le champ (grad $k V)$. Cette remarque permet de limiter les traitements numériques à des valeurs particulières de $U_{0}, q$ et $V$. On prendra en général $U_{0 \mathrm{n}}=10^{4} \mathrm{eV}$ et $q=1,6 \times 10^{-19} \mathrm{Cb}$.

Dans un système de coordonnées cylindriques $r$, $\theta, z$ (Fig. 1) les équations du mouvement s'écrivent :

$$
\left\{\begin{array}{l}
M \frac{\mathrm{d}^{2} r}{\mathrm{~d} t^{2}}-M r\left(\frac{\mathrm{d} \theta}{\mathrm{d} t}\right)^{2}=q E_{r}, \\
M \frac{\mathrm{d}}{\mathrm{d} t}\left(r^{2} \frac{\mathrm{d} \theta}{\mathrm{d} t}\right)=0, \\
M \frac{\mathrm{d}^{2} z}{\mathrm{~d} t^{2}}=q E_{z},
\end{array}\right.
$$

$E_{r}$ et $E_{z}$ sont les composantes du champ électrique interélectrode $\mathbf{E}$. Ces équations ne sont intégrables analytiquement que dans le cas des analyseurs sphé- riques (force centrale en $r^{-2}$ ). On sait qu'alors les trajectoires sont des ellipses [13] et [9].

On peut aborder le cas général de deux façons: chée,

- soit en utilisant une méthode analytique appro-

- soit en utilisant une méthode d'intégration numérique.

3.A MÉThode ANAlytiQue APPROChÉE. - Dans cette méthode, on suppose que les conditions de l'optique de Gauss sont remplies : pinceaux fins, ouverture angulaire petite, faible dispersion en énergie. On peut alors [15] résoudre analytiquement les équations du mouvement.

La trajectoire est définie par :

$$
\left\{\begin{array}{l}
\rho=\frac{\alpha_{0}}{\chi} \sin (\chi \theta)+\frac{2 \beta}{\chi^{2}}[1-\cos (\chi \theta)]+\rho_{0} \cos (\chi \theta), \\
\xi=\varphi_{0} \sqrt{\frac{R_{\mathrm{c}}}{r_{\mathrm{c}}}} \sin \left(\sqrt{\frac{r_{\mathrm{c}}}{R_{\mathrm{c}}}} \theta\right)+\xi_{0} \cos \left(\sqrt{\frac{r_{\mathrm{c}}}{R_{\mathrm{c}}}} \theta\right)
\end{array}\right.
$$

avec :

$$
\begin{gathered}
\chi^{2}=2-\frac{r_{\mathrm{c}}}{R_{\mathrm{c}}} \\
\rho=\frac{r}{r_{\mathrm{c}}}-1 \quad \text { et } \quad \xi=\frac{z}{r_{\mathrm{c}}}
\end{gathered}
$$

sont les coordonnées réduites du point courant d'une trajectoire définie par les paramètres initiaux suivants :

- $\beta$, écart de la vitesse initiale $v_{0}$ à la vitesse nominale $v_{0 \text { n }}$

$$
v_{0}=v_{0 \mathbf{n}}(1+\beta),
$$

- $\rho_{0}$ et $\xi_{0}$, valeurs de $\rho$ et $\xi$ à l'entrée,

- $\alpha_{0}$ et $\varphi_{0}$, angles d'incidence définis précédemment.

On remarque que dans les relations (2), les différents paramètres d'entrée sont séparés, de même que les paramètres du point courant $\rho$ et $\xi$. Ceci permet d'assimiler l'analyseur à un système optique à deux focales respectivement parallèle et perpendiculaire

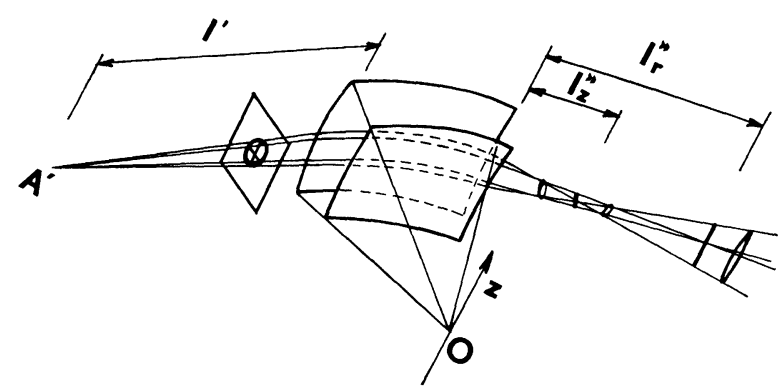

FIG. \$4. - Analyseur électrostatique, assimilé à un système optique à double focale. 
à l'axe $z z^{\prime}$ (Fig. 4), et de le traiter au moyen des formules classiques suivantes :

$$
\begin{aligned}
& \left(l^{\prime}-g_{r}\right)\left(l_{r}^{\prime \prime}-g_{r}\right)=f_{r}^{2} \\
& \left(l^{\prime}-g_{z}\right)\left(l_{z}^{\prime \prime}-g_{z}\right)=f_{z}^{2}
\end{aligned}
$$

avec

$$
\left\{\begin{array}{l}
g_{r}=\frac{r_{\mathrm{c}}}{\chi} \operatorname{cotg}\left(\chi \theta_{\mathrm{s}}\right), \\
g_{z}=\sqrt{r_{\mathrm{c}} R_{\mathrm{c}}} \operatorname{cotg}\left(\sqrt{\frac{r_{\mathrm{c}}}{R_{\mathrm{c}}}} \theta_{\mathrm{s}}\right), \\
f_{r}=\frac{r_{\mathrm{c}}}{\chi} \sin \left(\chi \theta_{\mathrm{s}}\right) \\
f_{z}=\frac{\sqrt{r_{\mathrm{c}} R_{\mathrm{c}}}}{\sin \left(\sqrt{\frac{r_{\mathrm{c}}}{R_{\mathrm{c}}}} \theta_{\mathrm{s}}\right)}
\end{array}\right.
$$

Cette formulation simple a l'avantage de faire apparaître le rôle que jouent les différents paramètres géométriques de l'analyseur $r_{\mathrm{c}}, R_{\mathrm{c}}, \theta_{\mathrm{s}}$. Elle permet, par un choix approprié de ces paramètres, de se placer dans un cas favorable à l'obtention de taux de comptage et de pouvoir de résolution donnés, tout en tenant compte d'éventuelles conditions sur les dimensions du détecteur.

En revanche, elle présente certaines insuffisances :

- les calculs ne sont valables qu'au premier ordre en $\rho, \xi, \beta, \alpha_{0}, \varphi_{0}$, et deviennent rapidement inextricables si l'on cherche à développer au second ordre ; cette limitation restreint la portée des ces calculs aux conditions de l'optique de Gauss, conditions qui cessent généralement d'être valables dans la réalité ;

- l'application des formules (2) au plan de sortie $\left(\theta=\theta_{\mathrm{s}}\right)$ se révèle peu réaliste lorsqu'on examine en détail les trajectoires à l'intérieur de l'analyseur. En appliquant les formules (2) à des plans méridiens successifs définis par leur angle au centre

$$
\theta_{n}=n \Delta \theta\left(0 \leqslant \theta_{n} \leqslant \theta_{\mathrm{s}}\right),
$$

on s'aperçoit qu'une fraction importante des particules qui atteignent théoriquement le détecteur franchissent en fait au cours de leur parcours les frontières $\mathrm{du}$ volume utile de l'analyseur (Fig. 5).

Ces constatations nous ont amenés à résoudre les équations générales du mouvement par une méthode purement numérique.

3. B MÉTHOde NUMÉRIQUE. - L'intégration numérique du système d'équations différentielles (1) est possible si l'on peut exprimer, en tout point $M$, le champ électrique $\mathbf{E}$ en fonction des coordonnées de ce point.

3.B.1 Expression du champ électrique $\mathbf{E}$ en fonction des coordonnées réduites. - Comme précédemment, on utilise les coordonnées réduites $\rho$ et $\xi$. $\mathrm{Si}$

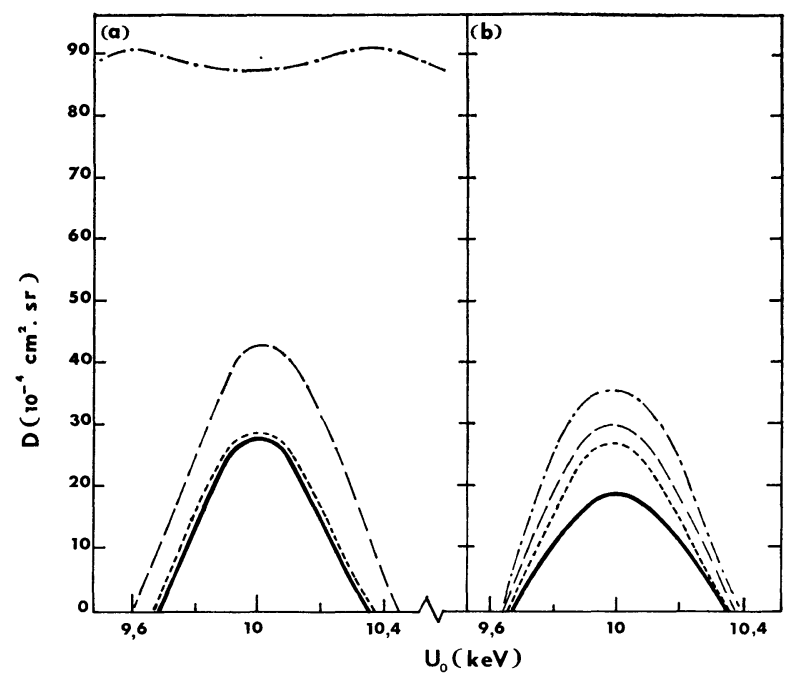

FIG. 5. - Comparaison des courbes $D\left(U_{0}\right)$ obtenues par différentes méthodes de calcul.

Paramètres géométriques : $r_{\mathrm{c}}=60 \mathrm{~mm} ; d=2,4 \mathrm{~mm}$;

$s=25 \mathrm{~mm} ; \theta_{\mathrm{s}}=127^{\circ} ; R_{\mathrm{c}}=120 \mathrm{~mm} ; z_{h}=\sigma=10 \mathrm{~mm}$.

Méthode analytique approchée,

- sans tenir compte des chocs $-\cdot-\cdot-$.

- en tenant compte des chocs

Méthode numérique,

$-\mathbf{E}=$ Cte :

- E obtenu par développements limités :

a) sans collimateur d'entrée,

b) avec collimateur : $h=35 \mathrm{~mm} ; d_{\mathrm{f}}=d ; \alpha_{\mathrm{m}}=0$.

$\Phi(r, z) \equiv \Psi(\rho, \xi)$ est le potentiel électrostatique entre les électrodes, on peut écrire :

$$
\left\{\begin{array}{l}
E_{r}=-\frac{\partial \Phi(r, z)}{\partial r}=-\frac{1}{r_{\mathrm{c}}} \frac{\partial \Psi(\rho, \xi)}{\partial \rho} \\
E_{z}=-\frac{\partial \Phi(r, z)}{\partial z}=-\frac{1}{r_{\mathrm{c}}} \frac{\partial \Psi(\rho, \xi)}{\partial \xi} .
\end{array}\right.
$$

Tenant compte de la symétrie par rapport au plan $z=0$, nous cherchons à exprimer la fonction $\Phi(r, z)$ sous la forme d'un développement en série entière des puissances paires de $z$ [16] :

$$
\Phi(r, z)=\sum_{v=0}^{\infty} \varphi_{v}(r) z^{2 v} .
$$

En écrivant que $\Phi(r, z)$ satisfait à l'équation de Laplace, $\nabla^{2} \Phi=0$, on obtient :

$$
\begin{aligned}
\sum_{v=0}^{\infty}\left[\frac{1}{r} \frac{\mathrm{d}}{\mathrm{d} r} \times\right. \\
\left.\times\left(r \frac{\mathrm{d} \varphi_{v}(r)}{\mathrm{d} r}\right)+(2 v+1)(2 v+2) \varphi_{v+1}(r)\right] z^{2 v}=0,
\end{aligned}
$$

ce qui fournit la relation de récurrence

$$
\varphi_{v}(r)=\frac{1}{(2 v) !}\left[-\frac{1}{r} \frac{\mathrm{d}}{\mathrm{d} r}\left(r \frac{\mathrm{d}}{\mathrm{d} r}\right)\right]^{v} \varphi_{0}(r) .
$$


Finalement, le problème se ramène à la détermination de la fonction $\varphi_{0}(r) \equiv \psi_{0}(\rho)$ que l'on cherchera à exprimer sous forme d'une série entière des puissances de $\rho$ :

$$
\psi_{0}(\rho) \equiv K \sum_{n=0}^{\infty} a_{n} \rho^{n}
$$

Pour obtenir les coefficients $a_{n}$, on exprime :

a) que le champ a la valeur $E_{0}$ sur l'équipotentielle moyenne $T\left(r=r_{\mathrm{c}}, z=0\right)$, sur laquelle on astreint le potentiel à être nul ;

b) que le potentiel est constant sur les électrodes.

La condition $a$ ) amène

$$
\psi_{0}(\rho)=-r_{\mathrm{c}} E_{0}\left[\rho+\sum_{n=2}^{\infty} a_{n} \rho^{n}\right]
$$

et l'expression du potentiel $\Psi(\rho, \xi)$ devient :

$$
\begin{aligned}
\Psi(\rho, \xi)=- & r_{\mathrm{c}} E_{0}\left\{\rho+\sum_{n=2}^{\infty} a_{n} \rho^{n}+\right. \\
+\sum_{v=1}^{\infty} \frac{(-1)^{v}}{(2 v) !} \xi^{2 v}[ & \left(\frac{1}{1+\rho} \frac{\mathrm{d}}{\mathrm{d} \rho}-\frac{\mathrm{d}^{2}}{\mathrm{~d} \rho^{2}}\right)^{v} \times \\
& \left.\left.\times\left(\rho+\sum_{n=2}^{\infty} a_{n} \rho^{n}\right)\right]\right\} .
\end{aligned}
$$

En pratique, on limitera ce développement à un ordre $n_{\mathrm{m}}$ compatible avec l'erreur maximale que l'on peut tolérer sur la valeur du champ $\mathbf{E}$ dans la résolution des éq. (1). Nous verrons ultérieurement comment déterminer cette valeur $n_{\mathrm{m}}$ que nous allons supposer connue pour l'instant. L'expression (5) peut alors s'écrire :

$$
\begin{aligned}
\Psi(\rho, \zeta)=-r_{\mathrm{c}} E_{0} & \left\{\sum_{k=1}^{k_{\mathrm{m}}} \Pi_{k} \xi^{2(k-1)} \times\right. \\
& \left.\times\left[\sum_{i=1}^{i_{k}}\left(\sum_{n=1}^{n_{\mathrm{m}}} \Gamma_{k, i, n} a_{n}\right) \rho^{i-1}\right]\right\}
\end{aligned}
$$

avec

$$
\left\{\begin{array}{l}
a_{1}=1, \\
k_{m}=\frac{n_{m}}{2}+1, \\
i_{k}=n_{m}-2 k+3 .
\end{array}\right.
$$

Les coefficients $\Pi_{k}$ et $\Gamma_{k, i, n}$ sont des coefficients numériques qui s'obtiennent au cours du passage de (5) à (6). Les coefficients $a_{n}$ dépendent des paramètres géométriques de l'analyseur et sont obtenus en exprimant la seconde condition $b$ ). Dans le cas traité ici, les équations des deux électrodes sont formellement identiques :

$$
\xi^{2}+(\rho+\tau)^{2}=(\tau+\varepsilon)^{2}
$$

où

$$
\left\{\begin{array}{l}
\tau=\frac{R_{\mathrm{c}}}{r_{\mathrm{c}}}, \\
\varepsilon=\left\{\begin{array}{l}
+\frac{d}{2 r_{\mathrm{c}}} \\
-\frac{d}{2 r_{\mathrm{c}}}
\end{array} \quad \text { pour l'électrode externe },\right.
\end{array}\right.
$$

Pour les géométries que nous avons adoptées, les valeurs de $d, R_{\mathrm{c}}$ et $r_{\mathrm{c}}$ sont telles que $d / R_{\mathrm{c}}=2 \varepsilon / \tau$ est du même ordre de grandeur que $d / r_{\mathrm{c}}=2 \varepsilon$, de telle sorte que nous pouvons limiter le développement de $\rho$ en fonction de $\varepsilon / \tau$ et $\xi$, infiniment petits de même ordre, de la même façon que le développement de $\Psi(\rho, \xi)$ précédent. Ceci permet d'écrire :

$$
\rho^{j}=\sum_{p=1}^{k_{\mathrm{m}}}\left[\sum_{q=1}^{q_{\mathrm{m}}} H_{j, p, q}\left(\frac{\varepsilon}{\tau}\right)^{q-1}\right] \xi^{2 p-2}
$$

où

$$
q_{\mathrm{m}}=n_{\mathrm{m}}+3-2 p .
$$

Alors la condition $\Psi(\rho, \xi)=$ Cte sur les électrodes devient :

$\Psi(\rho, \xi) \equiv \Psi_{1}\left(\xi, \frac{\varepsilon}{\tau}\right)=$

$=-r_{\mathrm{c}} E_{0}\left\{\sum_{k=1}^{k_{\mathrm{m}}} \Pi_{k} \xi^{2(k-1)}\left[\sum_{j=1}^{j_{\mathrm{m}}} A_{k, j}\left(\frac{\varepsilon}{\tau}\right)^{j-1}\right]\right\}=$ Cte où $j_{\mathrm{m}}=2 k_{\mathrm{m}}-2 k+1$ et $A_{k, j}$ est fonction linéaire de $H_{j, p, q}, \Gamma_{k, i, n}, a_{n}$.

Cette relation permet :

a) de calculer le potentiel à appliquer à chaque électrode en fonction de l'énergie nominale $U_{0 \text { n }}$ choisie :

$$
\Psi_{1}\left(0, \pm \frac{\varepsilon}{\tau}\right)=-r_{\mathrm{c}} E_{0} \pi_{1} \sum_{j=1}^{2 k_{m}-1} A_{1, j}\left(+\frac{\varepsilon}{\tau}\right)^{j-1}
$$

avec

$$
E_{0}=2 \mathrm{e} U_{0 \mathrm{n}} / q r_{\mathrm{c}}
$$

b) de déterminer le système d'équations linéaires qui fournit les $a_{n}$, en annulant les différents coefficients de $\xi^{2(k-1)}$ pour $2 \leqslant k \leqslant k_{\mathrm{m}-1}$. On dispose alors de $2\left(k_{\mathrm{m}}-2\right)=n_{\mathrm{m}}-2$ équations, une équation supplémentaire étant obtenue en annulant le terme $A_{k_{\mathrm{m}}-1}$. Ces $n_{\mathrm{m}}-1$ équations, dont on vérifie a posteriori l'indépendance, donnent la valeur des coefficients $a_{2}, \ldots, a_{n}$, puisque l'on connaît $a_{1}=1$.

On aura ainsi déterminé le potentiel électrostatique $\Psi(\rho, \xi)$ en tout point de l'analyseur, sous forme d'un développement en série des puissances de $\rho$ et $\xi$. La valeur du champ $\mathbf{E}(\rho, \xi)$ est obtenue immédiatement par dérivation de l'expression du potentiel.

Remarquons au passage que la méthode exposée ci-dessus n'est pas limitée au seul cas où les centres 
de courbure secondaires $\mathrm{O}_{a}$ et $\mathrm{O}_{b}$ sont confondus. Dans le cas de centres de courbure distincts, l'éq. (7) est remplacée par deux équations qui conduisent de manière analogue au système linéaire fournissant les valeurs de $a_{n}$.

3.B.2 Utilisation pratique de la méthode de calcul $d u$ champ. - Le calcul que nous venons de présenter est extrêmement lourd dans sa formulation générale, et d'application malaisée. Les résultats suivants nous ont permis de remédier à ces défauts.

a) Nous avons tout d'abord, pour une valeur assez élevée de $n_{\mathrm{m}}\left(n_{\mathrm{m}}=14\right)$, déterminé les coefficients $a_{n}$ en fonction des paramètres $\tau$ et $\varepsilon$, qui définissent la géométrie des électrodes (éq. (8)).

Ces coefficients $a_{n}(\tau, \varepsilon)$ ont les propriétés suivantes :

- Ils sont alternés : $a_{n}=(-1)^{n-1}\left|a_{n}\right|$.

- Leur valeur absolue décroît avec $\tau$ (Fig. 6a).

- Elle dépend peu de $\varepsilon$, de façon négligeable pour $\tau>1$ (Fig. 6b).

Nous avons d'autre part constaté que, dans un domaine très vaste $(0,01 \leqslant \varepsilon<0,1 ; 0,3 \leqslant \tau<10)$, les équipotentielles dans la section méridienne, sont très voisines de cercles de centre $O^{\prime}$ : pour l'angle $\omega_{0}$ (défini Fig. 1) inférieur à $\omega_{\mathrm{m}}=30^{\circ}$, la variation relative du champ le long de tels cercles est toujours inférieure à $10^{-2}$.

En pratique, les analyseurs toriques que nous étudions ont un volume utile tel que $\omega_{0}<\omega_{m}$, et on peut, dans ce volume, assimiler le champ à un champ radial.

On utilise alors en tout point, l'expression du champ obtenue dans le plan de symétrie :

$$
E_{\mathrm{s}}=E_{0}\left(1+\sum_{n=2}^{n_{\mathrm{m}}} n a_{n} \rho^{n-1}\right) .
$$

b) Nous avons cherché la valeur minimale de l'indice $n_{\mathrm{m}}$, qui permette de calculer les performances de l'analyseur avec la précision que l'on désire.

La valeur de $n_{\mathrm{m}}$ intervient de plusieurs façons :

- Elle fixe le nombre des termes du développement $\mathrm{du}$ potentiel; l'erreur qui en résulte sur le champ électrostatique $E_{\mathrm{s}}$ est de l'ordre de :

$$
\Delta E \simeq\left|E\left(n_{\mathrm{m}}\right)-E\left(n_{\mathrm{m}}+2\right)\right|,
$$

puisque les coefficients sont alternés.

- Elle détermine le système d'équations linéaires qui permet de calculer les $a_{n}$. La résolution sur calculateur de ce système entraîne une erreur qui est une fonction croissante de $n_{\mathrm{m}}$. En fait, pour tous les calculs que nous avons faits, cette erreur est négligeable devant $\Delta E$.

Nous avons fixé à $\Delta E / E=10^{-3}$ la limite tolérable en chaque point de l'erreur sur le champ $E_{\mathrm{s}}$ qui, intégrée le long des trajectoires, entraîne un déplacement du point de sortie de l'ordre de $0,1 \mathrm{~mm}$, ce qui est tout à fait négligeable.
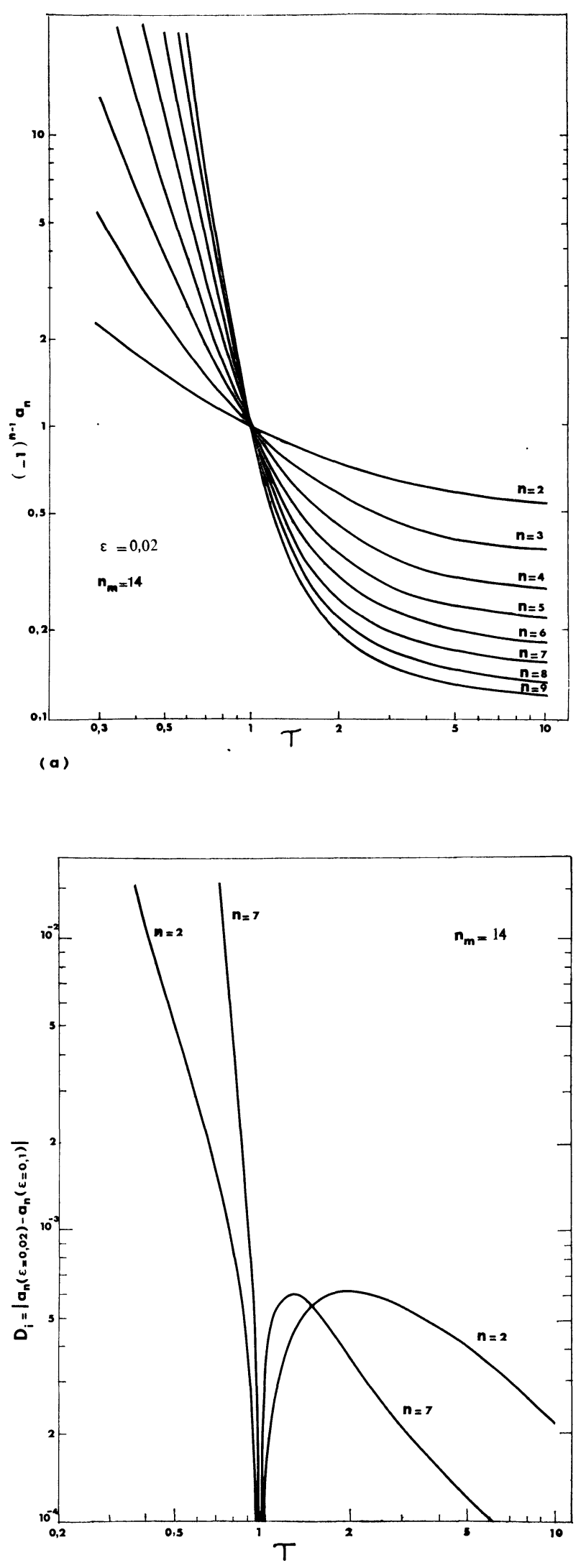

(b)

Fig. 6. - Evaluation du champ interélectrode par développements limités.

a) Coefficients $a_{n}$ en fonction de la courbure $\tau$.

b) Influence du paramètre $\varepsilon$ sur les coefficients $a_{n}$. 
La condition $\Delta E / E \leqslant 10^{-3}$ entraîne :

$$
n_{\mathrm{m}} a_{n_{\mathrm{m}}} \varepsilon^{n_{\mathrm{m}}-1} \leqslant 10^{-3} .
$$

Cette valeur de $n_{\mathrm{m}}$ est comprise entre 2 et 10 , suivant les valeurs de $\tau$ et $\varepsilon$ considérées.

3.B.3 Calcul des caractéristiques. - Ce calcul consiste à déterminer la trajectoire d'une particule de paramètres d'entrée $U_{0}, R_{0}, s_{0}, \alpha_{0}$ et $\varphi_{0}$ (Fig. 1 et 2) et à donner à une fonction $M\left(U_{0}, R_{0}, s_{0}, \alpha_{0}, \varphi_{0}\right)$ la valeur 1 ou 0 selon que la particule pénètre ou ne pénètre pas dans le détecteur. Le calcul de la trajectoire est fait par la méthode de Milne, dérivée de la méthode classique de Runge Kutta.

En faisant varier les 5 paramètres d'entrée, on décrit entièrement " l'espace objet ", les résultats étant présentés sous forme de tableaux (Fig. 8). Si les pas de variations des paramètres d'entrée sont respectivement $\Delta U_{0}, \Delta R_{0}, \Delta s_{0}, \Delta \alpha_{0}$ et $\Delta \varphi_{0}$, on obtiendra les grandeurs caractéristiques des analyseurs en calculant les expressions :

$$
\begin{aligned}
D_{1}\left(U_{0}\right)=\sum_{\alpha^{\prime}}^{\alpha^{\prime \prime}} \sum_{\varphi^{\prime}}^{\varphi^{\prime \prime}} \sum_{R_{\mathrm{a}}}^{R_{\mathrm{b}}} \sum_{s_{1}}^{s_{2}} M\left(U_{0}, R_{0}, s_{0}, \alpha_{0}, \varphi_{0}\right) \times \\
\quad \times Q\left(\alpha_{0}, \varphi_{0}\right) \Delta \alpha_{0} \Delta \varphi_{0} \Delta R_{0} \frac{R_{0}}{R_{\mathrm{c}}} \Delta s_{0} \\
F_{1}\left(\alpha_{0}\right)=\sum_{U^{\prime}}^{U^{\prime \prime}} \sum_{\varphi^{\prime}}^{\varphi^{\prime \prime}} \sum_{R_{\mathrm{a}}}^{R_{\mathrm{b}}} \sum_{s_{1}}^{s_{2}} M\left(U_{0}, R_{0}, s_{0}, \alpha_{0}, \varphi_{0}\right) \times \\
\times Q\left(\alpha_{0}, \varphi_{0}\right) \Delta U_{0} \Delta \varphi_{0} \Delta R_{0} \frac{R_{0}}{R_{\mathrm{c}}} \Delta s_{0} \\
G_{1}\left(\varphi_{0}\right)=\sum_{U^{\prime}} \sum_{\alpha^{\prime}}^{U^{\prime \prime}} \sum_{R_{\mathrm{a}}}^{\alpha_{\mathrm{b}}} \sum_{s_{1}}^{s_{2}} M\left(U_{0}, R_{0}, s_{0}, \alpha_{0}, \varphi_{0}\right) \times \\
\times Q\left(\alpha_{0}, \varphi_{0}\right) \Delta U_{0} \Delta \alpha_{0} \Delta R_{0} \frac{R_{0}}{R_{\mathrm{c}}} \Delta s_{0}
\end{aligned}
$$

où

$$
Q\left(\alpha_{0}, \varphi_{0}\right)=\cos ^{2} \varphi_{0} \cos \alpha_{0} .
$$

Dans le cas d'analyseurs avec collimateur à l'entrée ou fente de sortie, on garde le même programme général mais en annulant $M$ pour toutes les trajectoires incompatibles avec les diaphragmes.

Les erreurs qui interviennent dans la détermination des courbes de réponse ont deux origines :

- L'incertitude à l'endroit d'une frontière du domaine d'entrée provenant de la sommation de réponses discrètes que l'on peut évaluer statistiquement à $\sqrt{N^{\prime}} / 2 N, N^{\prime}$ étant le nombre de réponses nulles, $M=0$, voisines d'une réponse non nulle, $M=1$, et $N$ étant le nombre de réponses $M=1$.

\begin{tabular}{|c|c|c|c|c|c|c|}
\hline (c) $R_{b}+\frac{d}{4}$ & 列 & Dxt & $q_{x}$ & $\square$ & D & \\
\hline$k_{b}+\frac{d}{2}$ & $\begin{array}{l}10+3 \\
14-2\end{array}$ & 勧 & 洫 & 俚 & 且 & क \\
\hline$R_{b}+\frac{3 d}{4}$ & $\begin{array}{l}87 \\
12-2 y \\
16-9\end{array}$ & $\theta$ & $B$ & 戈 & 星 & 田 \\
\hline$R_{a}$ & 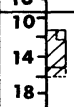 & 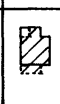 & 蚁: & 国。 & 降 & 诸 \\
\hline
\end{tabular}
On définit comme voisines deux réponses correspondant à des paramètres d'entrée tous identiques sauf un, valant $p$ dans un cas et $p \pm \Delta p$ dans l'autre.

- L'erreur faite dans l'exploitation graphique des courbes approchées $D_{1}\left(U_{0}\right)$, etc. Cette erreur

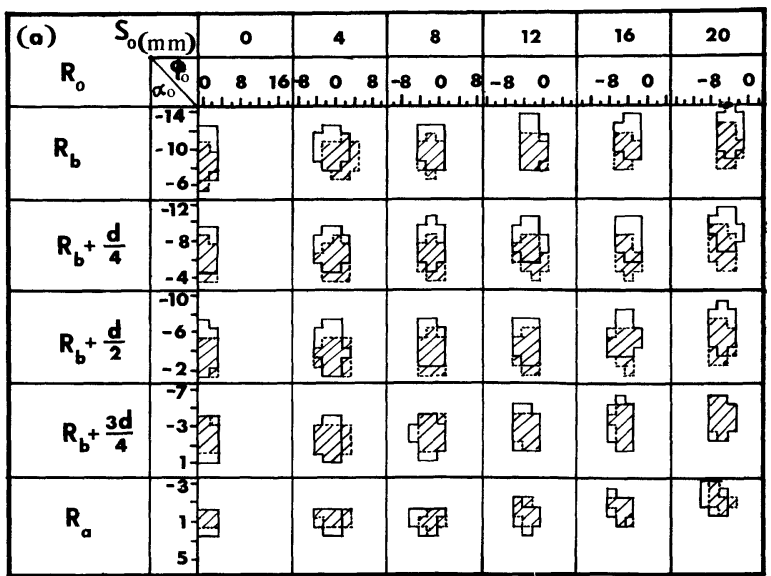

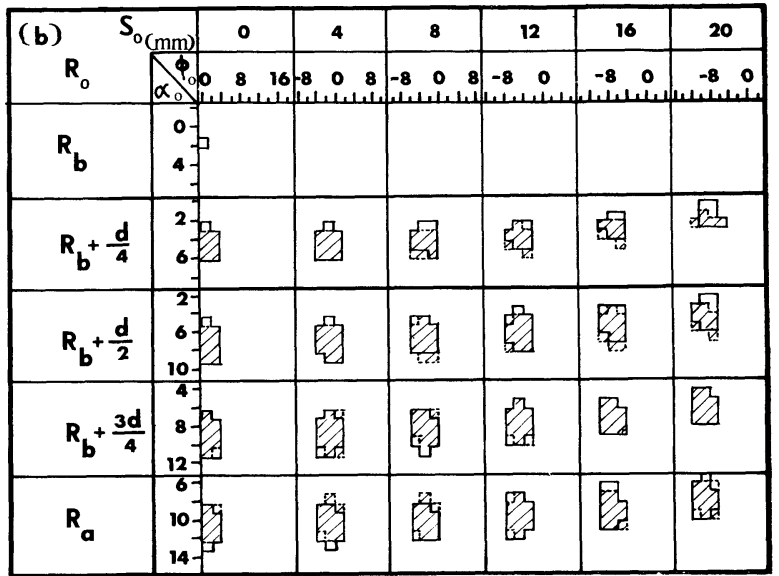

Fig. 8. - Réponses de l'analyseur.

Domaines tels que $M\left(U_{0}, R_{0}, s_{0}, \alpha_{0}, \varphi_{0}\right)=1$.

programme théorique,

[IIII] s simulation rhéographique,
a) $U_{0}=U_{\mathrm{b}}^{\prime}$,
b) $U_{0}=U_{1}$,
c) $U_{0}=U_{\mathrm{b}}^{\prime \prime}$.

dépend aussi de la forme des domaines d'entrée et des pas de variations $\Delta U_{0}$, etc.

On peut aboutir à une précision de l'ordre de 2 à $3 \%$, par l'utilisation d'un temps calcul suffisamment long ( $\simeq 20^{\prime}$ de IBM $360 / 65$, pour un cas de géométrie).

3.B.4 Comparaison avec les résultats obtenus par simulation en cuve rhéographique. - Pour nous assurer 
de la validité des calculs théoriques dans les zones suffisamment éloignées des bords de l'analyseur, et connaître l'importance des modifications du champ dans ces zones perturbées, nous avons procédé à l'étude en cuve rhéographique d'un analyseur particulier [17].

La maquette réalisée représentait, à l'échelle 10 , l'analyseur à large bande décrit au paragraphe 4.B. $\mathrm{Au}$ voisinage de l'entrée de l'analyseur, une électrode de correction a été placée, de manière à réduire l'influence, du champ de fuite (Fig. 7).

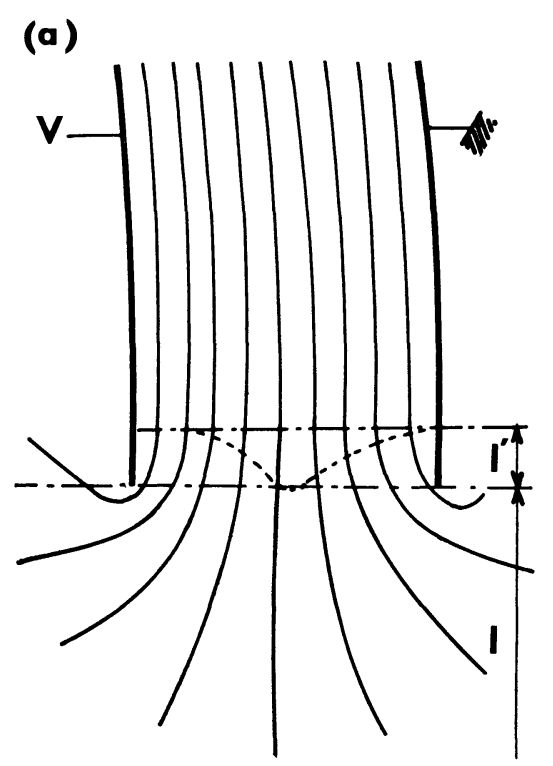

(b)

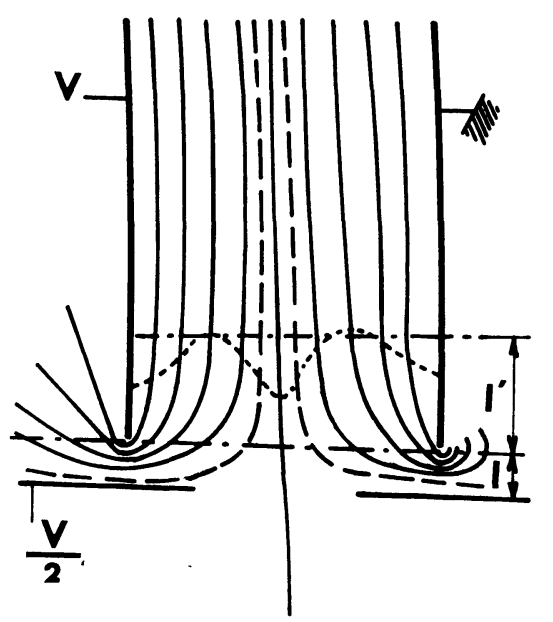

Fig. 7. - Allure des équipotentielles, observée par simulation rhéographique, dans le plan de symétrie, et au voisinage du plan d'entrée des électrodes.

traces des plaques et des électrodes,

équipotentielles $V_{k}=k(V / 10)$
$\ldots \ldots \ldots \ldots$

équipotentielles $V_{k}=k(V / 10)$,
équipotentielles intermédiaires,

....... limite de pénétration de la déformation,

a) sans électrode de correction $l=\infty ; l^{\prime}=18 \mathrm{~mm}$,

b) avec électrode de correction $l=10 \mathrm{~mm} ; l^{\prime}=32 \mathrm{~mm}$.
Les mesures du potentiel local ont été faites aux nœuds d'un réseau à trois dimensions. Pour calculer le champ électrostatique à partir du potentiel, nous avons lissé les valeurs du potentiel obtenues, ce qui conduit à une précision globale de l'ordre de $2 \%$ sur le champ.

Les trajectoires sont obtenues par l'application pas à pas de la loi $\mathbf{F}=q \mathbf{E}, \mathbf{E}$ étant supposé constant sur le pas d'intégration, et sa valeur obtenue par interpolation des valeurs $E_{x}, E_{y}, E_{z}$ aux nœuds du réseau de mesure.

Nous avons comparé les résultats bruts (réponses $\left.M\left(U_{0}, R_{0}, s_{0}, \alpha_{0}, \varphi_{0}\right)\right)$ avec ceux obtenus par la méthode purement numérique, pour 7 valeurs de l'énergie $U_{0}$. Trois de ces tableaux (les deux extrêmes, $U_{0} \simeq U^{\prime}{ }_{b}$ et $U_{0} \simeq U^{\prime \prime}{ }_{b}$, et le tableau central, $U_{0}=U_{1}$ ) sont portés sur la figure 8 .

Pour les valeurs extrêmes, on note une différence, faible mais significative, sur les angles $\alpha_{0}$ limites, et qui prend des valeurs opposées aux deux extrémités du domaine d'énergie. Nous pensons que ceci est la conséquence de la contraction des équipotentielles près des électrodes, au voisinage du plan d'entrée (voir Fig. 7). L'angle $\alpha$ limite est ainsi plus proche de zéro que ne le prévoit le programme théorique.

En revanche, pour les valeurs moyennes, les différences sont inférieures au pas du calcul, ce qui nous permet de vérifier par la même occasion que la précision de $2 \%$ des mesures en cuve rhéographique est suffisante.

Nous reproduisons sur les figures $9 b, c, d$, les courbes correspondant aux fonctions $D\left(U_{0}\right), F\left(\alpha_{0}\right)$, $G\left(\varphi_{0}\right)$, avec le collimateur indiqué au paragraphe 4. B. On remarquera l'excellente concordance des résultats expérimentaux et théoriques.

Pour montrer l'intérêt d'un développement à un ordre suffisant dans le calcul du champ (ici $n_{\mathrm{m}}=6$ ), nous donnons en figure $9 a$, les courbes $D\left(U_{0}\right)$ expérimentale, théorique avec le champ $E$ développé au $n_{\mathrm{m}}^{\text {ième }}$ ordre, et théorique avec l'approximation $E$ radial constant. Les courbes ont été tracées pour une collimation "idéale » $\left(h=\infty ;-1^{0} \leqslant \alpha_{0} \leqslant 11^{\circ}\right)$.

La méthode numérique complète se révèle bien adaptée à l'étude d'analyseurs réels, et nous allons en donner deux exemples d'application.

4. Application : performances de deux types de détecteurs destinés à des expériences spatiales. Ces détecteurs sont utilisés pour déterminer l'intensité, le spectre en énergie et la distribution angulaire des flux de protons et d'électrons dans certains domaines de l'environnement terrestre.

Selon les conditions et l'objectif de l'expérience, les performances que l'on vise sont très différentes. Ainsi que nous l'indiquerons dans les paragraphes suivants, on peut être amené à souhaiter une grande précision dans la détermination du spectre et de la distribution angulaire (recherche des mécanismes d'accélération pendant les sous-orages magnéto- 


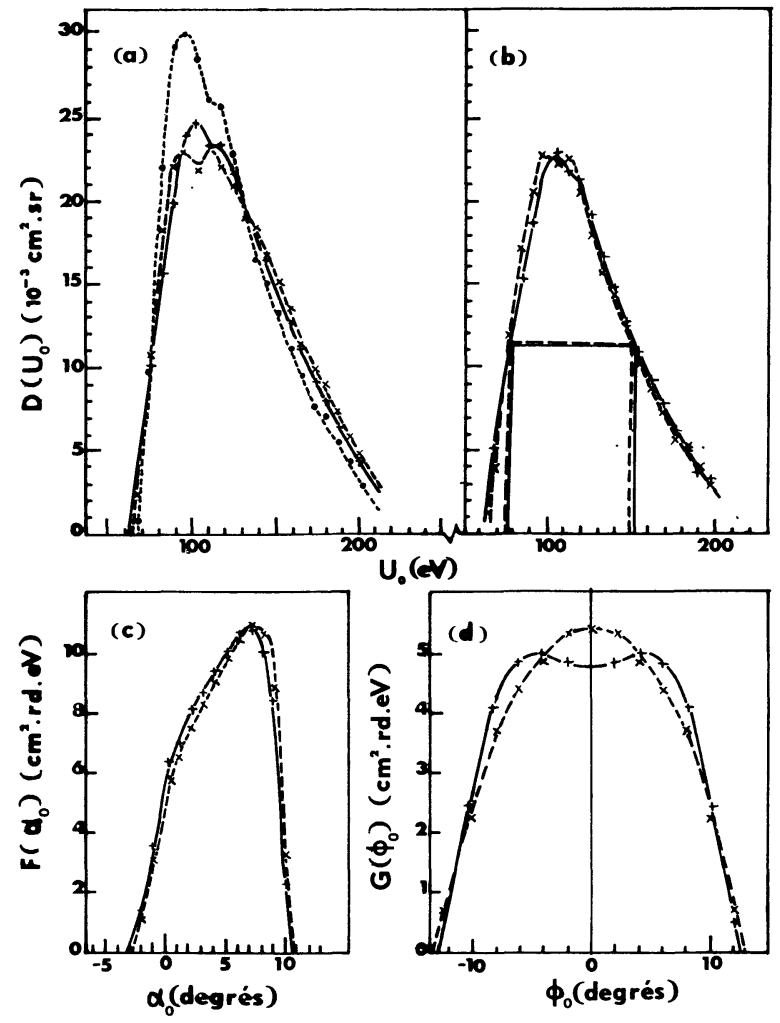

FIG 9. - Courbes de réponse de l'analyseur électrostatique à large bande.

- - - Champ expérimental.

- Méthode numérique complète.

. . . . . Méthode numérique avec $\mathbf{E}=$ Cte.

a) Sans collimation.

b), c), d) Avec collimation.

sphériques), ou bien à abandonner toute exigence dans ce domaine, pour obtenir un grand facteur de géométrie permettant la mesure de populations de particules dont les flux ont une très faible intensité.

Un certain nombre de contraintes dues aux conditions particulières de ce type d'expériences sont également à prendre en considération :

- pour des raisons de fiabilité évidentes, les hautes tensions d'analyse sont en général limitées à $5 \mathrm{kV}$ environ, ce qui impose une limite au rapport $d / r_{\mathrm{c}}$;

- étant donné le caractère transitoire des phénomènes que l'on peut être amené à détecter, un taux de comptage instantané suffisamment élevé pour être statistiquement valable est nécessaire. Par contre, le taux de comptage maximum qu'un multiplicateur d'électrons peut supporter est de l'ordre de $10^{5}$ coups par seconde, et l'on sait en outre que la durée de vie de ces capteurs est de l'ordre de $10^{11}$ impulsions. Ces deux contraintes opposées conduisent à rechercher des taux de comptage instantanés de l'ordre de $10^{2}$ à $10^{4}$ coups par seconde;

- les mouvements de rotation et de précession des véhicules spatiaux entraînent une variation de l'angle de visée du détecteur pendant la durée d'une mesure. Cette variation, dans le cas d'une fusée peut être de l'ordre de $3^{\circ}$. En outre, la précision de la restitution d'attitude d'un engin n'est guère supérieure à $\pm 2^{\circ}$. On comprend aisément qu'il est inutile de rechercher des résolutions angulaires meilleures que $3^{\circ}$ environ ;

- enfin, les critères d'encombrement et de poids limitent de façon notable les performances de ces détecteurs.

Nous avons entrepris une étude générale des performances des analyseurs toriques en fonction de leurs caractéristiques géométriques, afin de déterminer les solutions optimales correspondant à certains problèmes précis. Les résultats complets de cette étude feront l'objet d'un autre article, et nous nous contenterons de donner ici deux exemples particuliers d'utilisation de la méthode exposée au paragraphe 3 .

4.A ANAlyseurs a POUVOIR DE RÉSOlution Élevé. - Ces analyseurs sont destinés à des mesures en zone aurorale, dans une large gamme d'énergie : $10 \mathrm{eV}$ à $20 \mathrm{keV}$.

A l'intérieur de ce domaine, on prévoit une variation de flux très importante (de l'ordre de $10^{2}$ à $10^{3}$ ).

Nous sommes donc amenés à réaliser deux ensembles de détection, l'un réservé aux très basses énergies, de $10 \mathrm{eV}$ à $500 \mathrm{eV}$, l'autre aux énergies comprises entre $500 \mathrm{eV}$ et $20 \mathrm{keV}$.

Comme les multiplicateurs d'électrons n'ont une efficacité raisonnable qu'au-delà d'un seuil de $100 \mathrm{eV}$ environ, il faut prévoir pour les particules de basse énergie une accélération complémentaire, placée après l'analyseur électrostatique. Le faisceau accéléré ayant tendance à converger, il suffit de réduire légèrement la surface de la fente de sortie pour ne modifier aucune caractéristique.

On désire une bonne précision sur le spectre en énergie et la distribution angulaire : l'écart angulaire choisi est de $6^{\circ}$, et la résolution en énergie de $R_{\mathrm{u}} \simeq 5 \%$.

Afin d'obtenir un taux de comptage suffisant, compte tenu de ces forts pouvoirs de résolution, nous nous sommes placés dans le cas où le foyer image en $z$, de l'analyseur assimilé à un système optique, est placé au centre de la surface de détection. On peut en effet alors utiliser la surface d'entrée sur une grande dimension $s$, sans que direction et énergie d'incidence des particules détectées s'éloignent des conditions nominales.

Le multiplicateur tubulaire étant placé directement à la sortie de l'analyseur, cela correspond (voir éq. (3) et (4)) à la condition :

$$
\sqrt{\frac{r_{\mathrm{c}}}{R_{\mathrm{c}}}} \theta_{\mathrm{s}}=\frac{\pi}{2}
$$

Dans le cadre de cette hypothèse, nous avons pu montrer [17] que pour obtenir les résolutions ci-dessus, il fallait choisir un angle $\theta_{\mathrm{s}}$ assez grand. Nous avons adopté $\theta_{\mathrm{s}}=127^{\circ}$.

Les paramètres de collimation $h$ et $z_{h}$ sont choisis de telle façon que $\Delta \alpha \simeq \Delta \varphi \simeq A$, condition qui, 
nous l'avons montré, permet d'avoir un domaine $\mathrm{D}_{\mathrm{m}}$ maximum, pour chaque valeur donnée de $A$.

Enfin, les autres paramètres géométriques, $r_{\mathrm{c}}, d, s$, ont été fixés, compte tenu des valeurs de $D_{\mathrm{m}}$ et $R_{\mathrm{u}}$ demandées, en minimisant l'encombrement, tout en maintenant la tension d'alimentation inférieure à $4 \mathrm{kV}$.
Le tableau I présente, pour les deux valeurs extrêmes de $C$ demandées, les paramètres géométriques et les performances des analyseurs choisis.

A titre indicatif, il est intéressant de signaler qu'un analyseur sphérique ne donnerait qu'une résolution en énergie deux fois moins bonne, toutes conditions égales par ailleurs.

\section{TABLeAU I}

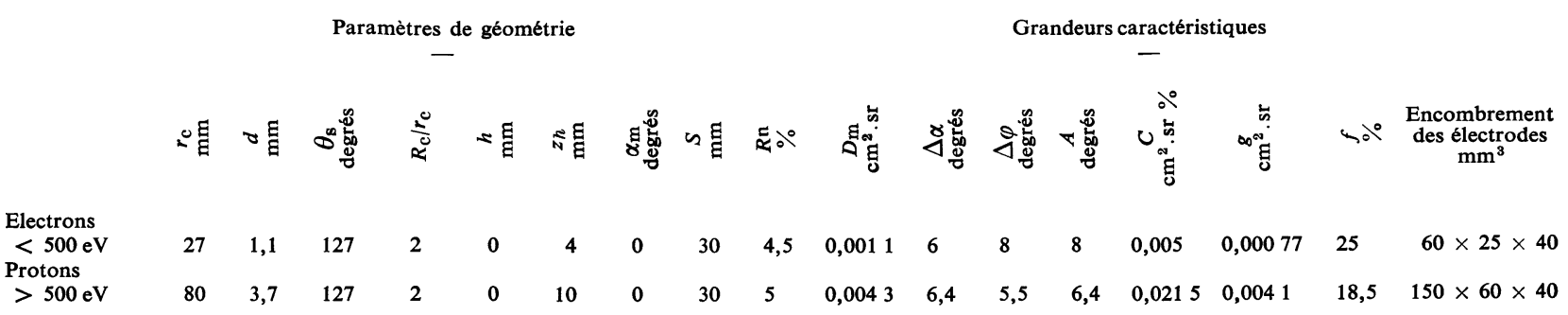

4. B Analyseur a LARge Bande passante. Cet analyseur est destiné à la mesure des flux de protons et d'électrons de basse énergie $(100 \mathrm{eV}$ à $35 \mathrm{keV}$ ), dans la zone de piégeage interne de la magnétosphère $(L<1,5)$.

Les flux sont pratiquement inconnus mais certainement faibles

$$
\left(J \simeq 10^{3} \text { à } 10^{5} \text { particules } . \mathrm{cm}^{-2} \cdot \mathrm{sr}^{-1} \cdot \mathrm{eV}^{-1} \cdot \mathrm{s}^{-1}\right),
$$

ce qui conduit à rechercher un fort domaine en énergie :

$$
C \simeq 0,5 \mathrm{~cm}^{2} . \mathrm{sr} \% \text {. }
$$

L'étude de la relation géométrie - performances de l'analyseur - nous indique que l'on ne peut atteindre cette valeur que pour une assez grande distance interélectrode $(d \simeq 8 \mathrm{~mm})$, et un faible angle de déviation $\left(\theta_{\mathrm{s}}<60^{\circ}\right)$, conditions qui correspondent à une valeur $R_{\mathrm{u}}$ grande ( $>40 \%$ ). On ne peut pas non plus atteindre de résolution angulaire très fine.

Nous avons alors choisi, plutôt que de faire des mesures ponctuelles, de faire des mesures sur des intervalles ayant une largeur $\Delta U_{n}$ proportionnelle à l'énergie moyenne $U_{n}$, tels que

$$
U_{n, k}\left(1+\frac{1}{2 k}\right)=U_{n, k+1}\left(1-\frac{1}{2 k}\right)
$$

$U_{n, k}$ et $U_{n, k+1}$ sont les énergies moyennes de deux intervalles jointifs; soit, pour 10 intervalles de mesure $\left(U_{n, 1}=100 \mathrm{eV}\right.$, et $\left.U_{n, 10}=35 \mathrm{keV}\right)$

$$
R_{\mathrm{u}}=\frac{100}{1,6}=65 \% \text {. }
$$

Il est souhaitable que l'écart angulaire $A$ ne dépasse pas $15^{\circ}$ environ.

Enfin, une tension d'alimentation maximale de $5 \mathrm{kV}$ fixe entraîne

$$
\frac{d}{r_{\mathrm{c}}} \leqslant 0,072
$$

Les équations analytiques au premier ordre, (3) et (4), permettent d'orienter le choix de la manière suivante :

Un encombrement raisonnable nous impose $r_{\mathrm{c}}<150 \mathrm{~mm}$. Compte tenu de $\theta_{\mathrm{s}}$ faible, cette valeur de $r_{\mathrm{c}}$ donnerait une valeur beaucoup trop faible de $R_{\mathrm{c}}$, si la relation (11) était vérifiée. On choisit alors de placer l'image $A_{r}^{\prime \prime}$ de la fente d'entrée dans le plan de sortie de l'analyseur, l'image $A_{z}^{\prime \prime}$ étant prise à l'infini (faisceau non divergent suivant l'axe $z^{\prime} z$, contrairement au cas des analyseurs cylindriques). Ces conditions, portées dans les éq. (3) donnent:

$$
\left\{\begin{array}{l}
l_{r}^{\prime \prime}=0, \quad \text { soit } \quad \sqrt{2-\frac{r_{\mathrm{c}}}{R_{\mathrm{c}}}} \theta_{\mathrm{s}}=k_{r} \pi, \\
l_{z}^{\prime \prime}=\infty .
\end{array}\right.
$$

Les seules solutions de ce système, compatibles avec les valeurs de $C$, de l'encombrement et de la tension maximum de polarisation, sont [17] :

$$
\theta_{\mathrm{s}} \simeq 30 \text { à } 40^{\circ}, \quad \frac{r_{\mathrm{c}}}{R_{\mathrm{c}}} \simeq 2
$$

La détermination exacte de la géométrie est alors obtenue comme suit :

a) La méthode numérique permet d'essayer un grand nombre de jeux de paramètres $\left(R_{\mathrm{c}}, r_{\mathrm{c}}, \theta_{\mathrm{s}}, d\right)$ autour des valeurs ci-dessus, et de les fixer de façon optimale :

$r_{\mathrm{c}}=120 \mathrm{~mm}, d=8,6 \mathrm{~mm}, \theta_{\mathrm{s}}=40^{\circ}, \frac{r_{\mathrm{c}}}{R_{\mathrm{c}}}=2,25$.

b) Nous avons ensuite étudié systématiquement l'influence de l'angle de visée $\alpha_{\mathrm{m}}$ et du collimateur sur les performances de l'instrument. Nous avons pu remarquer que $R_{\mathrm{u}}$ et $C$ augmentent lorsque l'on observe, non en incidence normale, mais autour de la direction $\alpha_{\mathrm{m}}=4^{\circ}$ à $5^{\circ}$. 
La distance $h$ de collimation a été choisie de manière à limiter $\Delta \alpha$ et à obtenir un bon facteur de forme $f$, sans encombrement prohibitif. La totalité de la surface de détection est utilisée $(\sigma=8 \mathrm{~mm})$.

Enfin, la dimension $s$ a été choisie en fonction des limites imposées à $A$.

En définitive, nous avons fixé :

$h=126 \mathrm{~mm}, \quad \alpha_{\mathrm{m}}=4,5^{\circ}, \quad d_{\mathrm{f}}=d, s=36 \mathrm{~mm}$.

c) Les performances finales sont les suivantes : $R_{\mathrm{u}}=(66 \pm 1,6) \%, A=17^{\circ} \quad\left(\right.$ avec $\left.\Delta \alpha=11^{\circ}\right)$ $\bar{g}=(1,77 \pm 0,04) \times 10^{-2} \mathrm{~cm}^{2} . \mathrm{sr}, f=20 \%$.

Ces performances ne pouvaient être obtenues, dans le cadre des contraintes imposées, à partir de formes simples d'électrodes. Pour un instrument cylindrique de mêmes dimensions, utilisé par Frank [2], la résolution est $R_{\mathrm{u}} \simeq 50 \%$, mais le facteur géométrique est très inférieur au nôtre : ramené à la même surface détectrice (en tenant compte de la répartition d'intensité dans le plan de sortie), le facteur de géométrie obtenu dans ce cas est environ 40 fois inférieur au nôtre.

5. Conclusion. - Des problèmes spécifiques de mesures en milieu spatial nous ont poussés à entreprendre l'étude d'analyseurs électrostatiques de forme torique.

Etant donné les conditions d'emploi (trajectoires non gaussiennes), la détermination précise des courbes de réponse de l'analyseur doit être faite par une méthode entièrement numérique. Le champ électrostatique est exprimé sous forme d'un développement limité, en fonction des coordonnées réduites d'un point, grandeurs qui restent petites dans le volume utile des électrodes. Le calcul, assez lourd dans sa généralité, peut être simplifié sans que sa précision soit affectée de façon notable, et il devient alors un outil efficace.

La bonne concordance des résultats obtenus, avec ceux que donne une méthode de détermination du champ électrostatique par simulation rhéographique, justifie l'emploi de la méthode numérique pour une étude précise du fonctionnement des analyseurs.

Les paramètres géométriques définissant la forme des électrodes et des systèmes de collimation sont nombreux, et ils influent de manière complexe sur les différentes grandeurs caractéristiques.

Nous avons cependant montré, sur deux exemples, que l'on pouvait faire choix d'analyseurs répondant de manière optimale à des problèmes de mesure donnés.

Ces choix ont été faits par l'utilisation de la méthode numérique, dans un cadre défini à partir des résultats d'une étude approchée, classiquement utilisée en spectrométrie de masse, et qui assimile l'analyseur à un système optique à deux focales.

Les performances obtenues pour ces exemples concrets, mettent en relief l'intérêt de la forme torique.

\section{Bibliographie}

[1] Birks (J. P.), Theory and practice of scintillation counting. Pergamon Press, 1964.

[2] Frank (L. A.), Univ. of Iowa Res. Rep., 65-22, 1965.

[3] Hoffman (R. A.), Evans (D. S.), J. Geophys. Res., $1968,73,6201$.

[4] Reme (H.), Thèse de Doctorat, 1969, Toulouse.

[5] Hughes (A. L.), Rojansky (V.), Physical Rev., 1929, 34, 284.

[6] Clarke (E. N.), Can. J. Phys., 1954, 32, 764.

[7] Marmet (P.), Kervin (L.), Can. J. Phys., 1960, 38, 787.

[8] Paolini (F. R.), Theodoridis (G. C.), Rev. Sci. Instr., 1967, 38, 579.

[9] Paolini (F. R.), Theodoridis (G. C.), Rev. Sci. Instr., 1968, 39, 326.
[10] Theodoridis (G. C.), Paolini (F. R.), Rev. Sci. Instr., 1969, 40, 621.

[11] Kerbele (A. N.), LASSettre (E. N.), The J. of Chem. Phys., 1964, 40, 271.

[12] Rogers (F. T. Jr), Rev. Sci. Instr., 1951, 22, 723.

[13] Ritchie (R. H.), CheKa (J. S.), Birkhoff (R. D.), Nucl. Instr. and Methods, 1960, 6, 157.

[14] Minkov (I. M.), Soviet Phys. Techn. Phys. (English Transl.), 1961, 5, 1285.

[15] Mc Dowell (C. A.), Mass spectrometry. Mc Graw Hill, 1963, 216.

[16] Svartholm (N.), Arkiv för Fysik, 1950, 2, 195.

[17] Decreau (P.), Prange (R.), Berthelier (J. J.), Note Technique GRI/NTP/88, 1971. 